\title{
Game-Based Learning Scenes Design for Individual User in the Ubiquitous Learning Environment
}

\author{
Stis $\mathrm{Wu}^{1}$, Maiga Chang ${ }^{2}$, and Jia-Sheng $\mathrm{Heh}^{1}$ \\ ${ }^{1}$ Dept. of Information and Computer Engineering, Chung-Yuan Christian Univ., Taiwan \\ stis@mcsl.ice.cycu.edu.tw, jsheh@ice.cycu.edu.tw \\ ${ }^{2}$ School of Computing and Information Systems, Athabasca University, Canada \\ maiga@ms2. hinet. net
}

\begin{abstract}
A ubiquitous learning environment provides learners opportunities of observing and touching the learning objects around the learners according to their preferences and/or interests. Learners can still solve problems, answer questions, and propose their own questions, in the ubiquitous learning environment. When we plan to make learners study in the ubiquitous learning environment, there are some issues needed to be taken into considerations, for examples, learners' interests and preferences. How to encourage learners' interests is an important issue, this paper designs and builds learning scenes to offer learners the personalized learning services based on game concepts. Each learning scene may cover one or many learning spots, and each learning spot has different learning objects. We can construct a series of learning scenes dynamically for individual learner based on the learner's choices, preferences and interests. Furthermore, a learning path involves learning scene switch is also generated automatically for the learner. Several exhibition rooms and artifacts in a museum are used to demonstrate the idea and mechanism proposed by this research.
\end{abstract}

Keywords: game-based learning, scene, ubiquitous learning, pervasive learning.

\section{Introduction}

In recent year, ubiquitous learning extends e-learning from indoor to outdoor, moreover, not like most of mobile learning applications which usually only provides the knowledge of single domain in particular environment, the ubiquitous learning emphasizes to offer learners interdisciplinary knowledge domains in the real world [1][4]. Mobile learning provides both teachers and learners a new learning way in the e-learning field, however, there is still an unsolved research issue, which is the flexible learning issue. The learners' learning activities will be limited in the specific learning environment and/or the specific domain knowledge arranged in advance. Ubiquitous learning not only extends e-learning from indoor to outdoor but also extends mobile learning from specific learning environment and specific knowledge domain to anyplace and multidiscipline [5].

Ubiquitous learning also provides the learning activities which allow learners to observe and touch the learning objects in the real world based on learners' choices and preferences. If learners want, they still can do suchlike solving problems, answering 
questions, expressing and organizing questions, and even brainstorming, during their learning processes [2][3].

Some researchers have thoughts that there are four characteristics of games could enhance learning effects: (1) challenges; (2) fantasy; (3) curiosity; and, (4) control [6]. Regarding the first characteristic, the challenges, Malone has offered the instructional activities which have a various difficulty levels to different learners according to learners' abilities [7][8]. The different learners then will face different challenges.

Active learning makes learners learning better because they are doing what they feel interesting and they feel that they are controlling everything. Games can encourage learners to learn after classes. Learners are usually 'actively' to play their favorite games. So, it might be work if we apply game concepts, scenes and scenes switching, to help learners 'active' learn and have fun in the ubiquitous learning environment.

The research analyzes and constructs learning scenes to offer learners personalized game-based learning services in a ubiquitous learning environment, for examples, a museum and a zoo.

According to personalized context-aware knowledge structure, different learning scenes can be extracted for different learners. Through personalized scenes construction, the learning service can extract the learner's learning preferences and design the learning path for individual learner.

Section 2 introduces the related works of ubiquitous learning environment, game-based learning, different knowledge structures, and different learning strategies. The relations and definitions between the elements of the learning scene are described in Section 3. Section 4 uses a way to construct learning scenes and introduces how to switch between the scenes. In Section 5, a real example of ubiquitous learning in the museum with learning scenes is built to demonstrate the effects of this research. Finally, Section 6 makes a conclusion and discusses the possible future works about individual scenes layout and switching.

\section{Scene Analysis}

\subsection{Scene Definitions}

For storing knowledge and learning object information, this research defines "learning scene" to record all learning locations and objects in the ubiquitous learning environment. Besides, the scene is also used to gather the information about what learning objects exist in different learning locations.

A scene will cover many learning areas; each learning area is a physical place, for example, an exhibition room in a museum. In each learning area, there is at least one or more learning spot. A learning spot may cover one or many learning objects. Take a museum for example, there are many exhibition rooms (the learning areas). Each room has at least one learning spots. When the visitor stands at each learning spot, he/she can see one or many artifacts (the learning objects).

There are two major elements in a scene as Figure 1 shows: (1) Learning spot (spot), indicates a learning location which covered the learning objects in a personalized knowledge structure; (2) Learning object (object), denotes possible learning objects for the learning spot. 


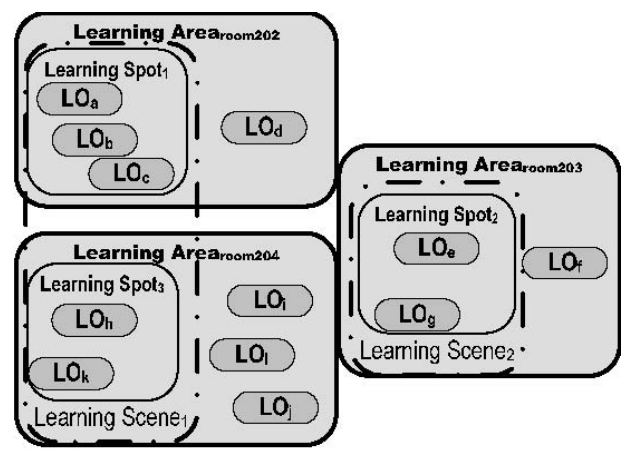

Fig. 1. Learining environment elements

\subsection{Relations between Scenes and Personalized Context-Aware Knowledge Structure}

The personalized context-aware knowledge structure then can be used to build learning scenes for learners. Because different learners with different preference will feel differently even when they are looking the same learning objects in the real world, hence, the suitable learning scenes to the learner can be built once the ubiquitous learning system has the personalized knowledge structure. Moreover, different learning activities can be given to learners depends on their preferences and learning objects' characteristics.

In Figure 2 there are two learners, Alex and Stis, have different viewpoints and/or preferences about the artifacts in the museum. The top part of Figure 2 shows Stis' personalized context-aware knowledge structure. Stis prefers the characteristic, "Dynasty", rather than "Color", hence, his personalized knowledge structure is constructed based on "Dynasty" characteristic. On the other hand, Alex has more interest in "Color", therefore his personalized knowledge structure root is constructed based on "Color" characteristic.

According to Wu's personalized knowledge structure [10], the scene subject for Stis is "Dynasty". The bottom part of Figure 2 shows how the ubiquitous learning system selects all learning objects related to the subject, "Dynasty", which Stis interests with to form a learning scene for Stis. Similarly, Alex's learning scene can be also constructed.

\subsection{Game-Based Measurement with Learning Scenes}

As the bottom part of Figure 2 shows, there are many learning objects matched the preference of a learner. The next problem is what learning object should the ubiquitous learning system suggests the learner learning and observing first.

Figure 3 shows an example of two learning scenes cover three learning spots and some learning objects. Assuming a learner stands at the learning spot2. At learning spot2, the learner can find two learning objects, $\{\mathrm{LO} 2, \mathrm{LO} 3\}$. Each learning object has its own characteristics about "Dynasty" subject. For example, the learning object LO2 may have characteristic suchlike "Items of daily use were specially produced for the Ming imperial court". Moreover, the degree of realizing these story-like characteristics 


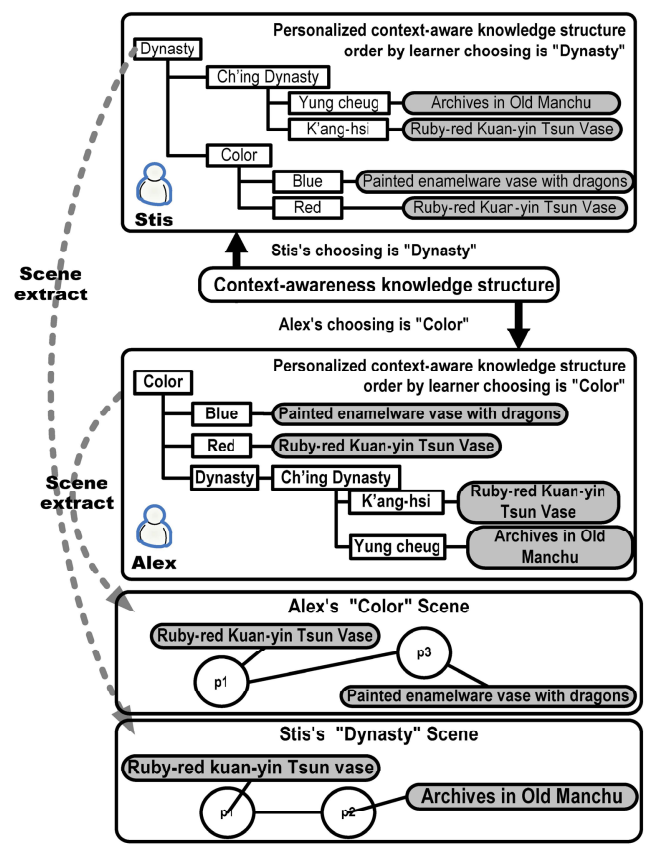

Fig. 2. Two personalized context-awareness knowledge structures

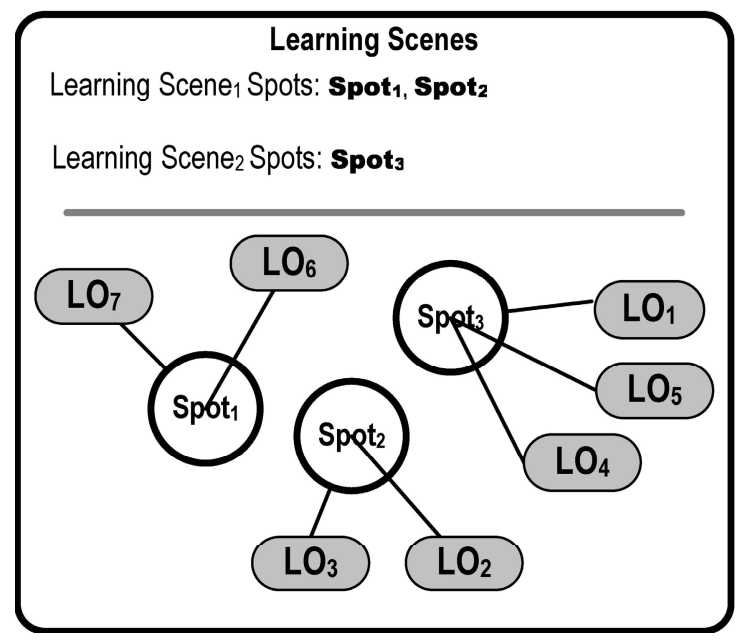

Fig. 3. Example of learning scene and objects

of learning objects can be seen as a quantitative measurement of learners' abilities and/or skill points based on the game-based learning theory.

This research defines the game-based measurement with the personalized learning scenes based on the probability theory. If a learner gets the idea from the questions, then the probability of specific characteristic will be raised and the probability 
represent the learner's degree of mastery in the specific characteristic. When the overall probability of a learning scene achieved a threshold, the ubiquitous learning system can say that the learner clears the stage or levels up, and ready to challenge another stage or level (another learning scene).

\section{Scenes Construction and Switching}

\subsection{Scenes Construction}

This research develops a way to extract scenes from the personalized knowledge structure. The whole scene construction flow involves 5 phases as Figure 4 shows:

Phase I: Learning materials analysis, the learning objects in real environment have a lot of information (or also called characteristics), which might cover different domains or places. In this phase, we need to analyze the learning objects and its characteristics in the real world first. After this phase, the context-aware knowledge structure can be built.

Phase II: Basic personalized context-aware knowledge structure construction, because the personalized context-aware knowledge structure should be created according to the learners' preferences and interests. The basic personalized context-aware knowledge structure for individual learner can be constructed depends on which stories the learners interest with.

Phase III: Personalized context-aware knowledge structure refinement, even if two learners choose the same story in phase II, their interests still might be a little different. In order to precisely refine the personalized context-aware knowledge structure, the system asks the learner some advance questions about the characteristics of the learning objects which are involved in the story the learner has chosen. The personalized context knowledge structure is then refined according to the learner's feedback.

Phase IV: Personalized context-aware knowledge structure generation, after repeating the phase II and III, the correspondent learning objects and characteristics the learner might need and/or interest with are clear to the system. The learner's personalized context-aware knowledge structure is then can be generated in this phase.

Phase V: Learning scenes construction, the ubiquitous learning system can extract the learning objects that the learner may prefer according the personalized context-aware knowledge structure. Because the personalized knowledge structure can represent different knowledge domain and the learning objects locate at different locations, the ubiquitous learning system then construct the game-based learning scenes based on the distinction features of the selected learning objects, such as "location" and "domain", as Figure 4 shows.

\subsection{Scenes Switching Principles}

This research uses rough set theory [9] to develop scene switching methodology. In the rough set theory, data will be divided into three sets, including the Positive Set (POS), the Negative Set (NEG), and one or more Boundary Sets (BNDs). This research defines the positive set data as the learning objects which have more than one characteristic the 


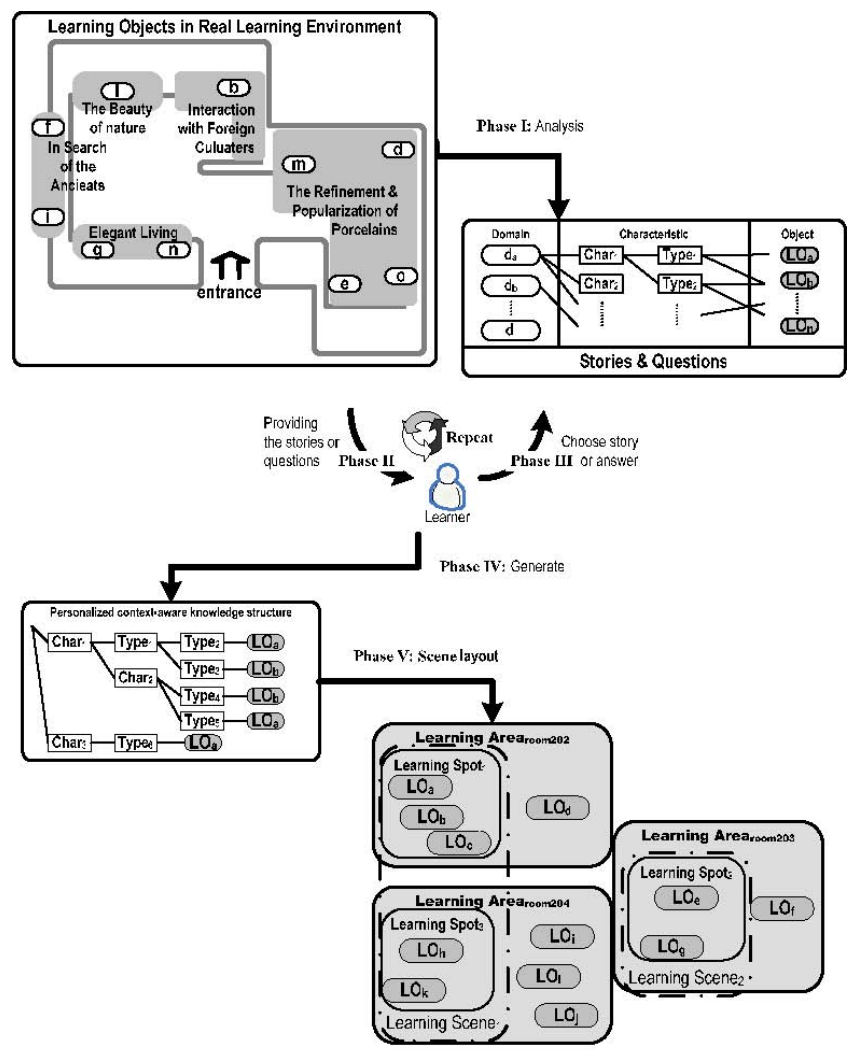

Fig. 4. The five phases to build individual scene

learner is interesting with, the learner has to observe the learning objects in the positive set. On the contrary, the learning objects in the negative set don't have any characteristic that the learner interest with. Unlike the positive set and the negative set, there are many boundary sets in the learning scene. The learning objects in a boundary set also have the characteristics that the learner interests with. The difference between the positive set and the boundary set is that all of the learning objects in the same boundary set have the same characteristics, and the learner only needs to observe one of the learning objects for the same boundary set when he/she does learning activities in the ubiquitous learning environment.

Figure 5 shows an example of using rough set to categorize learning objects involved in learning spots. At top part of Figure 5, there are two learning scenes cover three learning areas. Each learning scene has one or more learning spots, and each learning spot contains one or more learning objects. In the middle part of Figure 5, every learning object has many characteristics. The learning objects can be categorized into three sets. Table 1 lists a summary of the sets and its learning objects.

From Table 1 the ubiquitous learning system knows that the learner has to observe the learning object LOa. If the scene only includes four characteristics, including 


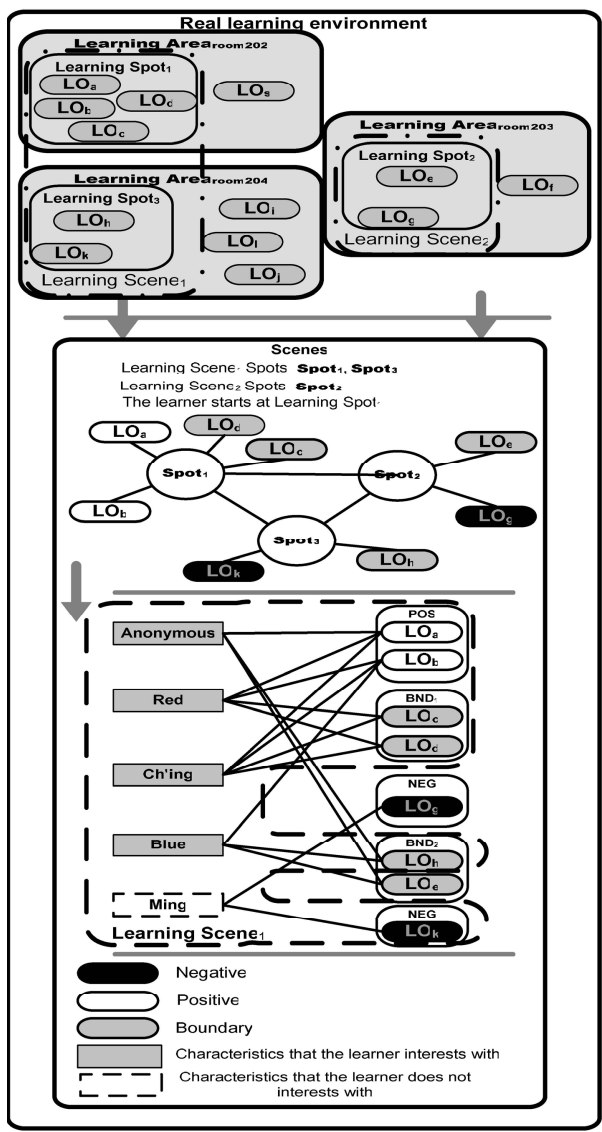

Fig. 5. The relation between characters and learning objects

Table 1. Three sets and its learning objects

\begin{tabular}{|l|l|}
\hline POS & $\mathrm{LO}_{\mathrm{a}}, \mathrm{LO}_{\mathrm{b}}$ \\
\hline $\mathrm{NEG}$ & $\mathrm{LO}_{\mathrm{g}}, \mathrm{LO}_{\mathrm{k}}$ \\
\hline $\mathrm{BND}_{1}$ & $\mathrm{LO}_{\mathrm{c}}, \mathrm{LO}_{\mathrm{d}}$ \\
\hline $\mathrm{BND}_{2}$ & $\mathrm{LO}_{e}, \mathrm{LO}_{\mathrm{h}}$ \\
\hline
\end{tabular}

"Anonymous", "Red", "Ch'ing", and "Blue", then the ubiquitous learning system can do scene switching after the learner finished observing the LOa and LOb.

\section{Complete Example}

\subsection{Game-Based Learning in Museum}

By using the game concept to help learner learning in the ubiquitous learning environment, the first thing is to define the learner profile suchlike "ID", "ability", and 
"major preference". The major preference includes the characteristics in the personalized context-aware knowledge structure suchlike "dynasty", "color", "function", and "author", in Figure 7. Ability is the learning object characteristics which learner has been observed. ID is the learner's name.

\subsection{Scenario}

This paper takes learning in a museum for example. In the museum, there are many artifacts with different subjects and/or topics exhibiting in the different rooms. For example, the Room 203 as Figure 6 shows, the "gray area" represents different subjects and/or topics (learning spots) suchlike "Elegant Living", "In Search of the Ancients", and "The Beauty of Nature"; "black circle" represents the artifacts (the learning objects) in the room; the number is the artifacts number. There are eleven learning objects in Room 203. This research considers a room as a learning area.

According the Phase I in Figure 4, Figure 7 shows the analysis results of learning objects. Figure 7 represents how to use context-aware knowledge structure to store a museum's learning objects and its characteristics.

The personalized context-aware knowledge structures and game-based learning scenes are constructed based on with the learners' choices and their answers about the questions of artifacts' characteristics as Figure 8 shows. The width or depth of personalize context-aware knowledge structure depends on how much the learners' interest with and how exquisite they know.

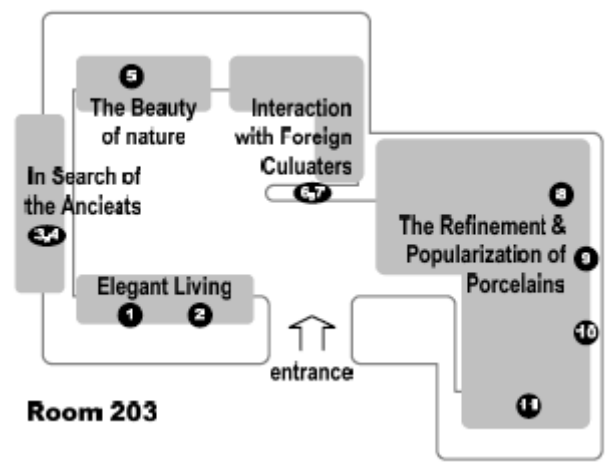

Fig. 6. Room 203 in a museum

Figure 9 shows the process of constructing the learning scenes after the learner answered the questions. After the learner answered the questions, the ubiquitous learning system can revise the personalized context-aware knowledge structure and find the preference learning objects and interesting characteristics from the personalized context-aware knowledge structure. Furthermore, using the personalized knowledge structure to realize the learning preference objects' locations, defining the learning spots, and constructing the learning scenes for individual learner as the bottom part of Figure 9 shows. 


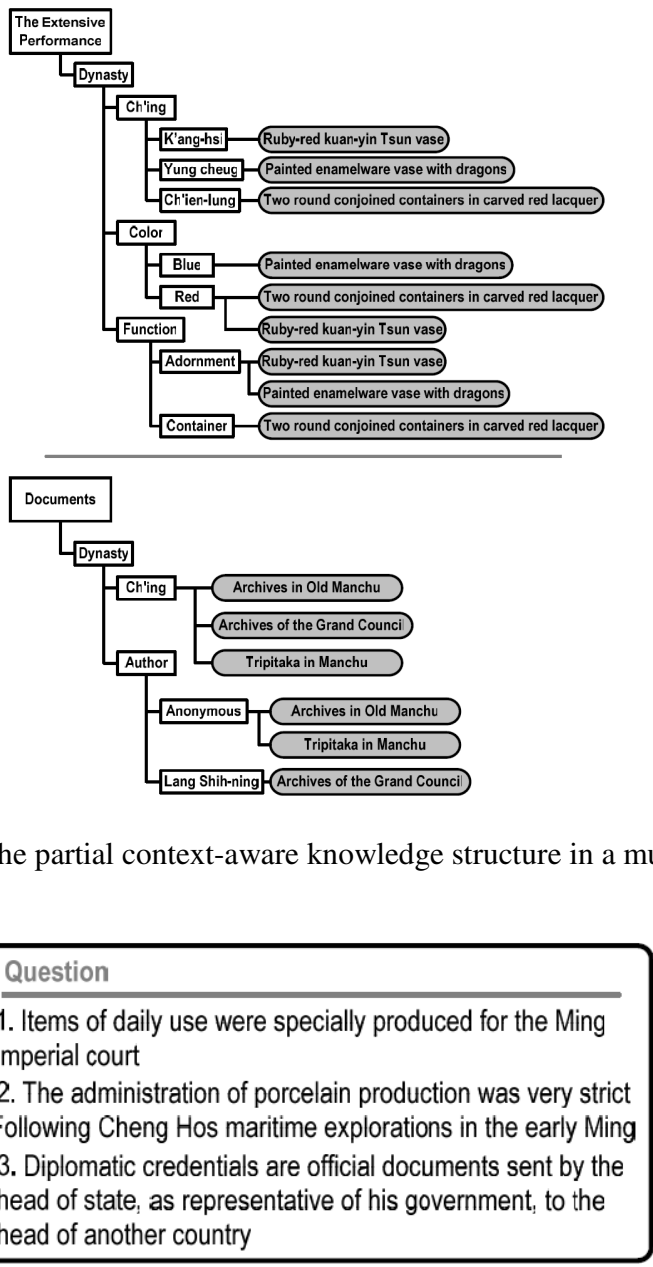

Fig. 8. The questions about "The Fashionable vs. the Antiquarian"

\subsection{Individual Learning Path in Museum}

With the learning scenes, this research uses the distances to plan the observation sequence for the learning objects in the positive set and boundary set that the learner may interest with.

The ubiquitous learning system then first picks the learning objects in the POS set, including "Ruby-red kuan-yin Tsun vase", "Two round conjoined containers in carved red lacquer", "Tripitaka in Manchu", "Archives of the Grand Council", and "Archives in Old Manchu". The learning path has to route around the POS set learning objects.

Figure 10 uses the "white ellipse" to represent the learning objects in the POS set, the must be observed learning objects. The scene can be switched from the Function Scene to the Document Scene after the learner has finished observing the white ellipse 


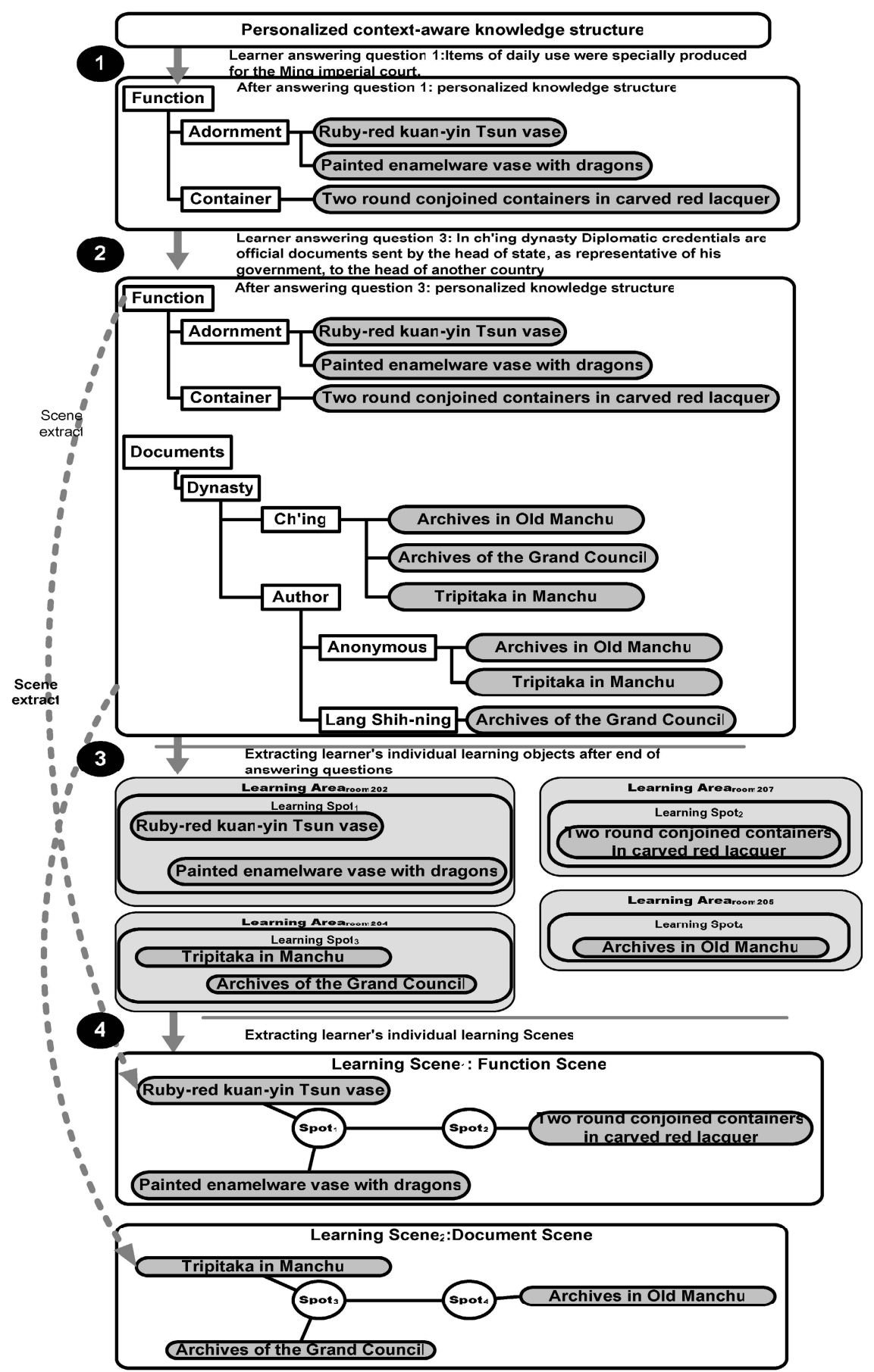

Fig. 9. Scenario constructing process 

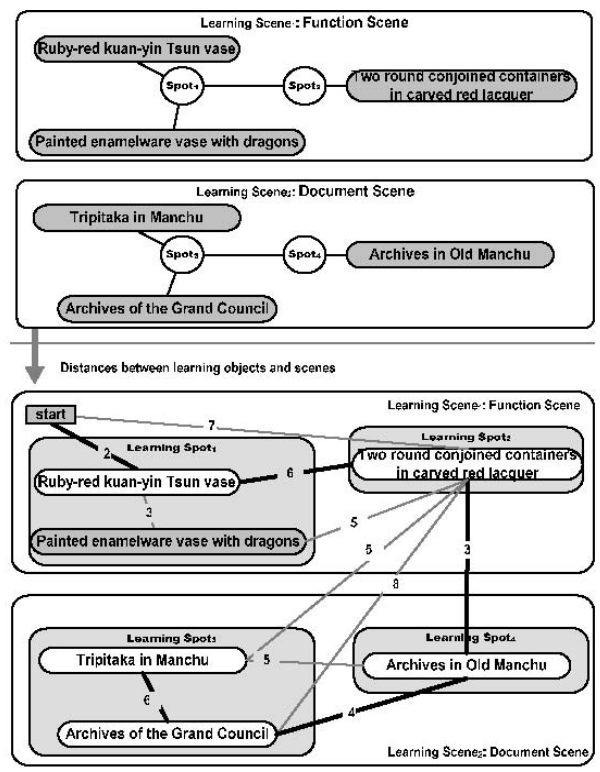

Fig. 10. Function scene and document scene relationship in scenario

learning objects. According to the distance, the ubiquitous learning system generates the learning guidance path: start Æ"Ruby-red kuan-yin Tsun vase" Æ"Two round conjoined containers in carved red lacquer" $Æ " A r c h i v e s$ in Old Manchu" $Æ " A r c h i v e s$ of the Grand Council" Æ"Tripitaka in Manchu".

\section{Conclusions}

There are still some research issues could be discussed and done in order to improve the scene switching and learning path generation. For example, as we mentioned, the challenge issue. Currently, the game-based ubiquitous learning system uses probability as the measurement of learner (player) abilities. The next step is how to provide an automatically generated and non-interrupt adaptive test for such kind of ubiquitous learning environment. Regarding the learning path generation issue, the current research and system uses only distance between the learning objects. As we all know, it is just the simplest solving way, in the future research, other factors suchlike learning scenes should be taken into consideration.

\section{References}

[1] Chang, A., Chang, M.: Adaptive Mobile Navigation Paths for Elementary School Students' Remedial Studying. In: Proceedings of the IEEE International Conference on Interactive Computer Aided Learning (ICL 2006), Villach, Austria, September 27-29 (2006) 
[2] El-Bishouty, M.M., Ogata, H., Yano, Y.: Personalized Knowledge Awareness Map in Computer Supported Ubiquitous Learning. In: Proceedings of the 6th IEEE International Conference on Advanced Learning Technologies (ICALT 2006), Kerkrade, The Netherlands, July 2006, pp. 817-821 (2006)

[3] Felder, R.M., Brent, R.: Learning by Doing. Chemical Engineering Education 37(4), 282-283 (2003), Retrieved on January 15, 2008, from http: / www4 .ncsu.edu/ unity/lockers/users/f/felder/public/Columns/Active.pdf

[4] Hwang, G.-J.: Criteria and Strategies of Ubiquitous Learning. In: Proceedings of the IEEE International Conference on Sensor Networks, Ubiquitous, and Trustworthy Computing (SUTC 2006), Taichung, Taiwan, June 2006, pp. 72-77 (2006)

[5] Hall, T., Bannon, L.: Designing ubiquitous computing to enhance children's learning in museums. Journal of Computer Assisted Learning 22(4), 231-243 (2006)

[6] Lepper, M.R., Malone, T.W.: Intrinsic motivation and instructional effectiveness in computer-based education. Aptitude, Learning, and Instruction, III: Conative and Affective Process Analysis, pp. 255-286. Erlbaum, Hillsdale, New Jersey (1987)

[7] Malone, T.W.: Toward a theory of intrinsically motivating instruction. Cognitive Science 5(4), 333-369 (1981)

[8] Malone, T.W., Lepper, M.R.: Making learning fun: A taxonomy of intrinsic motivations for learning. Aptitude, Learning, and Instruction, III: Conative and Affective Process Analysis, pp. 223-253. Lawrence Erlbaum Associates, Hillsdale (1987)

[9] Pawlak, Z.: Rough Sets: Theoretical Aspects of Reasoning about Data. Kluwer Academic Publishers, Norwell, MA

[10] Wu, S., Chang, A., Chang, M., Liu, T.-C., Heh, J.-S.: Identifying Personalized Context-aware Knowledge Structure for Individual User in Ubiquitous Learning Environment. In: Proceedings of the 5th IEEE International Conference on Wireless, Mobile, and Ubiquitous Technologies in Education (WMUTE 2008), Beijing, China, March 23-26, 2008 (in press, 2008) 